\title{
développement des essais en centrifugeuse en Chine
}

\author{
development of centrifuge testing in China
}

WEI-XIN ZHU

Institut de Recherche Hydraulique de Nanjing (R.P. Chine)*

Rev. Franç. Géotech. n 48 , pp. $43-48$ (juillet 1989)

\section{Résumé}

La première étude sur modèle réduit d'un problème géotechnique faite en chine remonte à 1981 . Depuis lors la modélisation en centrifugeuse connaît un développement significatif. L'article décrit les cinq plus importantes installations en service et dresse la liste de l'ensemble des problèmes étudiés en centrifugeuse.

\section{Abstract}

The first centrifugal study of a geotechnical problem in China was made in 1981. Since then, centrifugal modelling has known a significant development. The paper presents the five largest facilities in service and gives a list of all the problems studied with a centrifuge. 


\section{INTRODUCTION}

La Chine, avec une superficie de 9,6 millions de $\mathrm{km}^{2}$, est au troisième rang après l'URSS et le Canada. Grand pays en développement, elle assure depuis 1980 la modernisation des grands secteurs de son économie. L.es grands ouvrages de génie civil sont nombreux et variés en nature. Les investissements consacrés aux travaux relevant de la géotechnique représentent une part importante des capitaux consacrés à la construction. De plus, les projets sont souvent implantés dans des sites où les risques naturels sont sérieux tels que tremblements de terre, tempétes, ce qui représente un défi supplémentaire pour le géotechnicien. C'est pourquoi la Chine attache-t-elle de l'attention au progrès dans les études en géotechnique.

Les calculs précis des ouvrages en terre et de leurs fondations présentent des difficultés certaines dues à la non linéarité de la relation contrainte - déformation et à la variabilité en fonction du temps des caractéristiques mécaniques des sols. Ainsi, les nombreuses méthodes analytiques ou empiriques ne peuvent être considérées que comme des méthodes approchées.

Actuellement, pour l'édification des grands ouvrages de géotechnique, trois approches sont employées: la modélisation numérique, la modélisation physique et lobservation de l'ouvrage. Les informations tirées des études seront répercutées vers la conception, la construction et les travaux d'entretien pour obtenir économie et sécurité des projets.

Pour développer l'agriculture et résoudre le problème essentiel qui est de nourrir un milliard d'habitants, environ cent mille réservoirs ont été créés en Chine au cours des quarante dernières années et ce, pour une large part, avec des barrages en terre et enrochements. Le Ministère des Ressources en Eau et de l'Energie Electrique (MREEE) attache ainsi de l'importance à la géotechnique car cette discipline traite de la sécurité et de l'économie des travaux hydrauliques. C'est pourquoi, la majorité des spécifications techniques en géotechnique et des appareillages d'essais des sols, proviennent en Chine du MREEE. C'est aussi ce ministère qui représente la Chine dans toutes les activités du comité ISO/TC-182.

\section{L'ORIGINE DE LA MODÉLISATION GÉOTECHNIQUE EN CENTRIFUGEUSE EN CHINE}

Depuis longtemps, il y avait plus de 10 centrifugeuses employées pour les études sur modèles de structures avec la photoélasticité. Dans les années 60 , sous influence venue d'URSS, quelques chercheurs commencèrent à s'intéresser, en Chine, à l'utilisation en géotechnique des modèles réduits centrifugés. Les informations sur ce sujet ont été traduites du russe en chinois. Cependant, c'est seulement dans les années 80 que l'on a vraiment porté attention à la modélisation en centrifugeuse, en Chine, sous linfluence des travaux de Grande-Bretagne, des Etats-
Unis, du Japon, etc. En particulier, le professeur WENG-XI HUANG de l'Université Tsing Hua, qui est un pionnier dans le développement des modèles numériques en géotechnique, a défendu la modélisation en centrifugeuse après une visite des Universités de Cambridge et de Manchester effectuée en 1980. Le MREEE appuya I'Institut de Recherche Hydraulique de Nanjing (IRHN) pour développer les essais en centrifugeuse pour la géotechnique. Une centrifugeuse servant aux études en photoélasticité à l'Université de Hehai fut modifiée pour les applications géotechniques par l'IRHN dès 1981. Une vue en est donnée par la figure 1. Pour cette centrifugeuse modifiée, de nombreux appareillages de mesure furent développés, du personnel technique fut formé à cette modélisation physique et quelques problèmes de recherche appliquée furent rapidement correctement résolus. Par exemple, en 1982-83, l'IRHN employa la modélisation en centrifugeuse pour étudier avec succès la rupture d'un mur de quai, afin d'en connaître le mécanisme (ZHU et al., 1988).

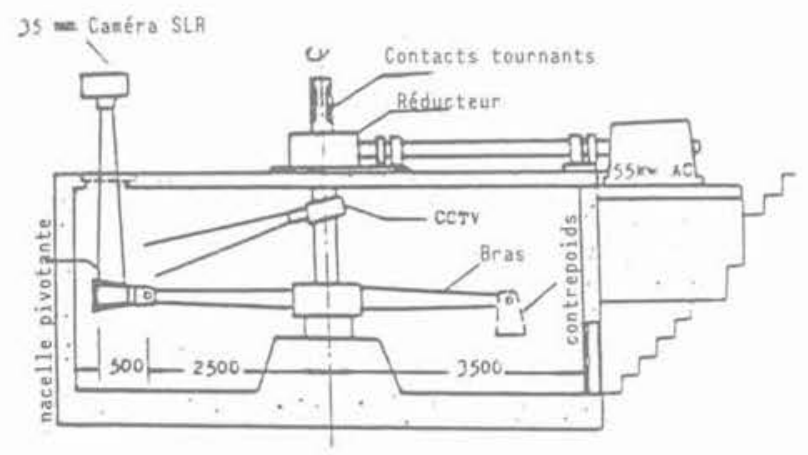

Fig. 1. - Centrifugeuse de I'IRHN.

Fig. 1. - Centrifuge of the Nanjing Hydraulic Research Institute.

Les résultats obtenus sur modèle réduit s'avérèrent conformes au mode de rupture de l'ouvrage du site. Par conséquent, il fut possible d'établir les causes de l'accident, ce que les autres méthodes n'avaient pas permis de faire. Ceci fut la première application de la modélisation en centrifugeuse pour résoudre un problème pratique en Chine, et ce fut fait comme le veut la maxime chinoise: "triompher dès la première bataille». Cette première application attira plus encore l'attention du MREEE qui envoya en 1984 une nouvelle délégation conduite par les professeurs WENGXI HUANG et WEI-XIN ZHU au Etats-Unis pour y étudier les centrifugeuses, les techniques de modélisation et les applications en géotechnique afin d'examiner l'intérêt et la faisabilité de la construction d'une grande centrifugeuse pour la géotechnique, en Chine.

A la fin de 1984, une proposition fut présentée par le professeur ZHU. Celle-ci fut acceptée à l'unanimité des experts chinois qui s'accordèrent sur la nécessité de construire une grande centrifugeuse et non de petites machines destinées aux recherches de base et à l'éducation des étudiants. Depuis lors, il y eut cinq projets et deux grandes centrifugeuses pour la géotechnique sont en construction et devraient être achevées en 1990. De plus, trois centrifugeuses, petites ou moyennes, sont en fabrication et devraient être en 
service en 1989 à l'IRHN, à l'Université des Sciences et de la Technologie de Chengdu et à l'Université des Communications du Sud-Ouest.

Afin d'apprendre les nouvelles techniques de modélisation en centrifugeuse le plus rapidement possible, de nombreux instituts et universités chinois, envoient des personnels techniques à l'étranger.

\section{LES CENTRIFUGEUSES}

Sur les cinq centrifugeuses existant en Chine, trois servent à la géotechnique et aux études en photoélasticité (fig. 1, 2, 3). La centrifugeuse de l'Institut Scientifique de Recherche de la Rivière Yangtze fut construite en 1983 (WANG, 1988) ; c'est la plus grande des centrifugeuses chinoises existantes. La figure 4 présente une petite centrifugeuse installée en 1987 à l'Institut des Chemins de Fer de Shanghaï. La centrifugeuse de Chengdu est la modification d'un simulateur de vol capable de $55 \mathrm{~g}$ (fig. 5) (LIU et TANG, 1988).

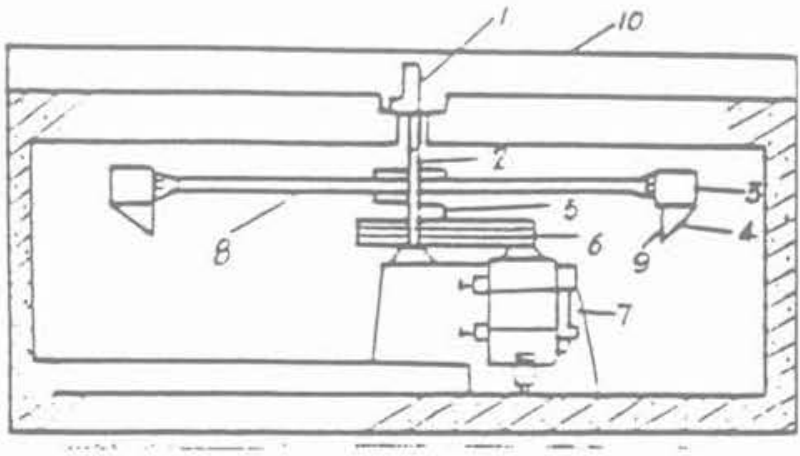

Fig. 2. - Centrifugeuse de I'Université Huhai (25 tonnes-g).

1. Contacts tournants - 2. Arbre - 3. Nacelle pivotante

4. Miroir - 5. Caméra TV - 6. Courroie - 7. Moteur

8. Rotor - 9. Cadre - 10. Plaque de couverture

Fig. 2 - Centrifuge of Huhai University (25 g-tons)

1. Sliprings - 2. Shaft - 3. Swinging basket

4. Mirror - 5. TV camera - 6. Belt - 7. Motor

8. Rotor - 9. Frame - 10. Cover

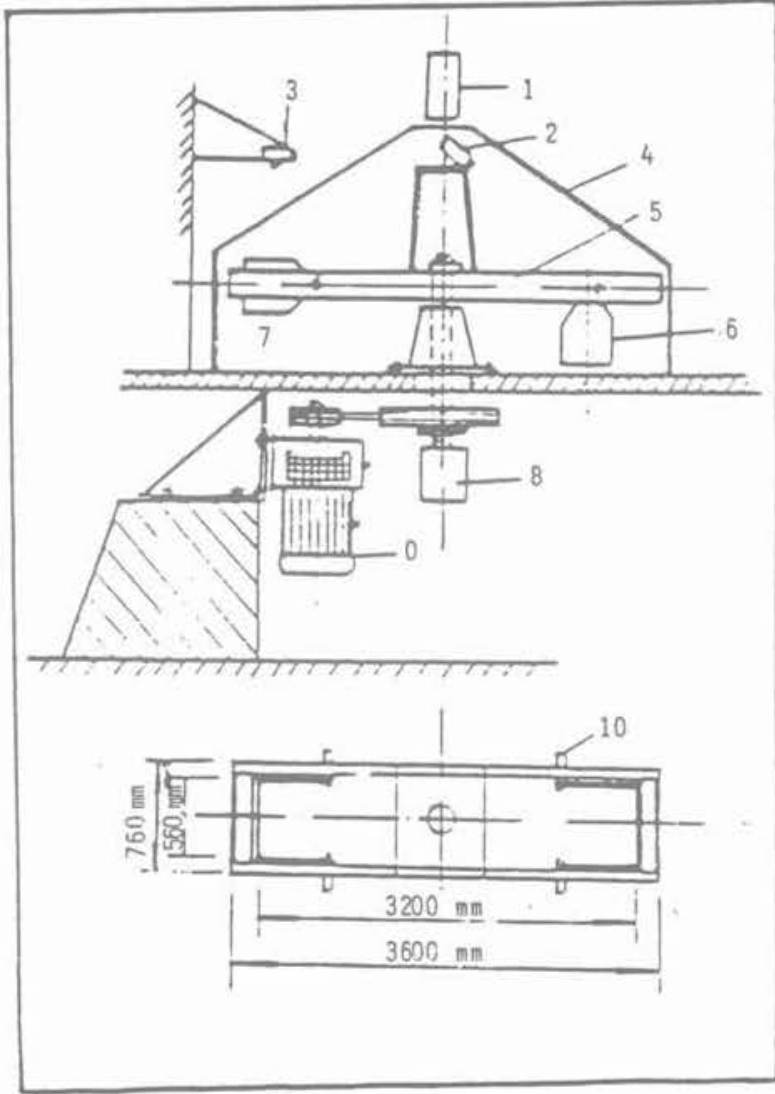

Fig. 4. - Centrifugeuse de PICFS.

1. Contacts tournants - 2. Camera TV - 3. Appareil photo 4. Enceinte - 5. Rotor - 6. Nacelle pivotante à l'arrêt

7. Nacelle pivotante en rotation - 8. Joint tournant hydraulique 9. Moteur $(15 \mathrm{~kW})$ - 10. Barre de torsion

Fig. 4 - Centrifuge of the Shanghai Railways Institute 1. Sliprings - 2. TV Camera - 3. Still camera

4. Enclosure - 5. Rotor - 6. Swinging basket at stop 7. Swinging basket in rotation - 8. Hydraulic slipring 9. Motor $(15 \mathrm{~kW})-10$. Torsion bar

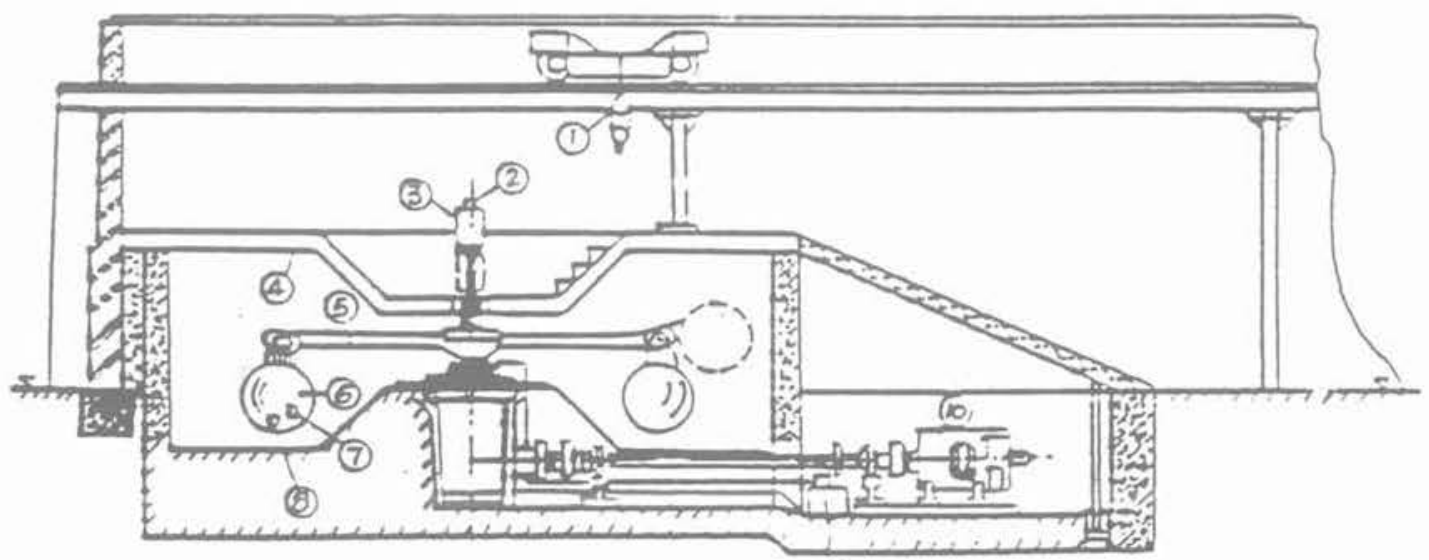

(9)

Fig. 3. - Centrifugeuse de I'IRSRY 1300 tonnes-g).

1. Pont roulant - 2. Système optique - 3. Contacts tournants - 4. Plafond - 5. Rotor - 6. Nacelle pivotante 7. Camera TV - 8. Appareil photo - 9. Réservoir d'huile - 10. Moteur courant continu

Fig. 3. - Centrifuge of the Yangtze River Scientific Research Institute (300 g-tons).

1. Travelling crane - 2. Optical system - 3. Sliprings - 4. Ceiling - 5. Rotor - 6. Swinging basket

7. TV Camera - 8. Still camera -9. Oil tank - 10. D.C. Motor 


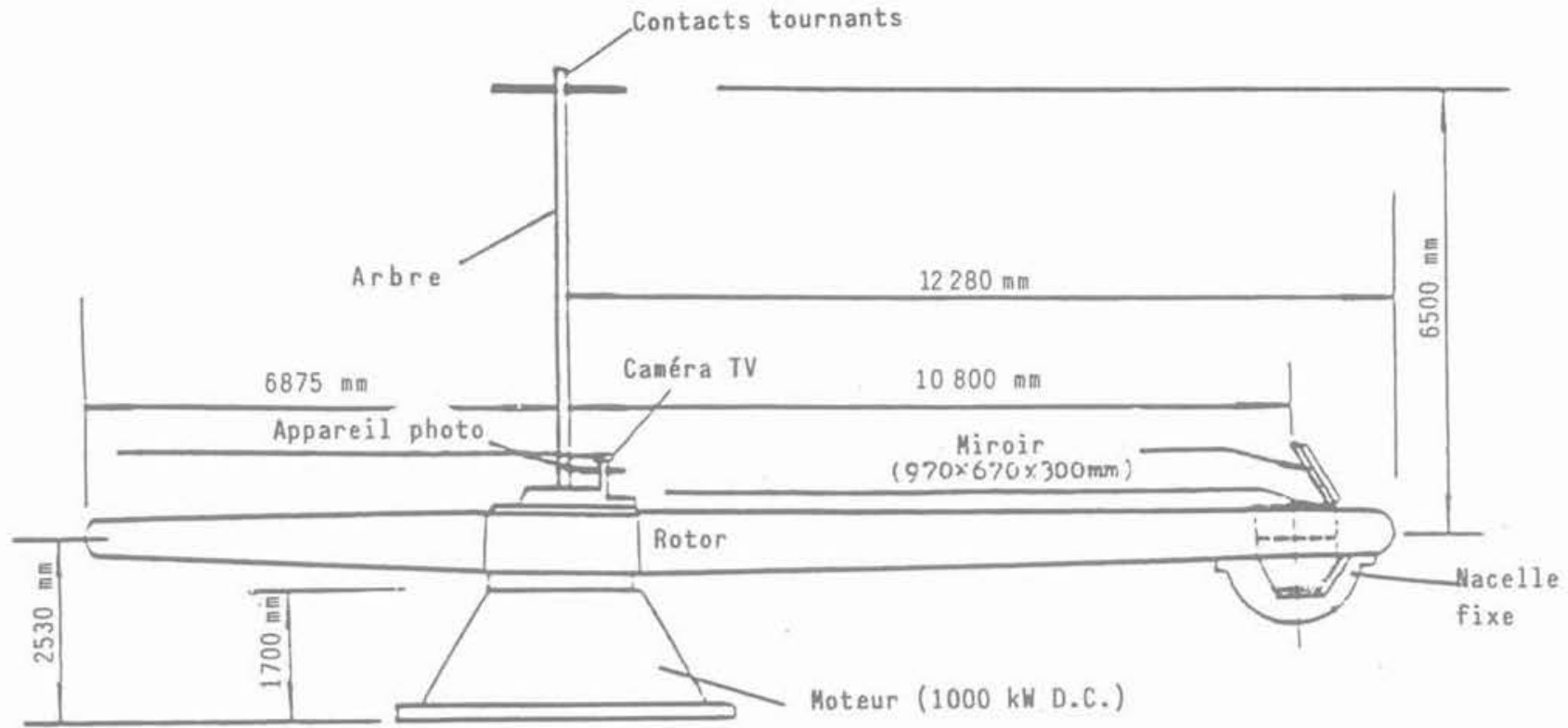

Fig. 5 - Centrifugeuse de I'IPEHC (330 tonnes-g).

Fig. 5. - Centrifuge of the Chengdu Hydroelectric Design and Research Institute (330 g-tons)

Les caractéristiques des deux grandes centrifugeuses en projet (JIA et al., 1988, ZHU et LIU, 1988) sont résumées dans le tableau 1 avec celles des autres machines.

Tableau 1. - Spécifications techniques des centrifugeuses géotechniques chinoises.

Table 1. - Technical specifications of the Chinese geotechnical centrifuges

\begin{tabular}{|c|c|c|c|c|c|c|c|}
\hline Lieu & \multicolumn{2}{|c|}{$\begin{array}{l}\text { Institut de } \\
\text { Recherche } \\
\text { Hydraulique } \\
\text { de Nanjing } \\
\text { IRHN }\end{array}$} & $\begin{array}{l}\text { Institut de } \\
\text { Recherche } \\
\text { Scientifique } \\
\text { de la rivière } \\
\text { Yangtze } \\
\text { IRSRY }\end{array}$ & $\begin{array}{l}\text { Institut de } \\
\text { conception et } \\
\text { d'études } \\
\text { Hydroélectrique } \\
\text { de Chengdu } \\
\text { ICENC }\end{array}$ & $\begin{array}{l}\text { Université } \\
\text { de Hehai } \\
\text { UH }\end{array}$ & $\begin{array}{l}\text { Institut } \\
\text { des Chemins } \\
\text { de fer } \\
\text { de Shanghai } \\
\text { ICFS }\end{array}$ & $\begin{array}{c}\text { Institut } \\
\text { de Conservation } \\
\text { de I'Eau et } \\
\text { de Recherche } \\
\text { Hydroélectrique } \\
\text { ICERH }\end{array}$ \\
\hline $\begin{array}{l}\text { Rayon effectif } \\
\text { max. (m) }\end{array}$ & 2,9 & 5,0 & 3,0 & 10,8 & 2,4 & 1,55 & 4 \\
\hline $\begin{array}{l}\text { Accélération } \\
\text { max. (g) }\end{array}$ & 200 & 200 & 300 & 110 & 250 & 200 & 300 \\
\hline $\begin{array}{l}\text { Masse modèle } \\
\text { max. }(\mathrm{kg})\end{array}$ & 100 & 2000 & 1000 & 3000 & 100 & 100 & 1500 \\
\hline $\begin{array}{l}\text { Capacité max } \\
\text { (tonne-g) }\end{array}$ & 20 & 400 & 300 & 330 & 25 & 20 & 450 \\
\hline $\begin{array}{l}\text { Puissance moteur } \\
\text { (KW) }\end{array}$ & 55 & 500 & 400 & 1000 & 40 & 15 & 700 \\
\hline $\begin{array}{l}\text { Années premières } \\
\text { études en } \\
\text { géotechnique }\end{array}$ & 1982 & 1990 & 1985 & 1985 & 1982 & 1987 & 1990 \\
\hline Figure $n^{\circ}$ & 1 & & 3 & 5 & 2 & 4 & \\
\hline
\end{tabular}




\section{ACTIVITÉS DE RECHERCHE EN MODÉLISATION EN CENTRIFUGEUSE}

Un résumé chronologique (1982-1988) est donné dans le tableau 2. Les études indiquées ont été faites pour l'essentiel en vue de résoudre des problèmes urgents de conception ou de construction. Comme la modélisation géotechnique en centrifugeuse n'est uti- lisée en Chine que depuis moins de 10 ans, aucune installation ou matériel n'est idéal, et tous les problèmes traités se rapportent à des applications simples statiques. Les limites des centrifugeuses existantes rendent difficile létude de problèmes complexes incluant des effets dynamiques. C'est pourquoi, cinq nouvelles centrifugeuses, grandes, moyennes et petites, dédiées aux études géotechniques seront construites d'ici 1990.

Tableau 2. - Liste des études géotechniques en centrifugeuse à l'institut de Recherche Hydraulique de Nanjing. Table 2. - List of the geotechnical centrifuge studies at the Nanjing Hydraulic Research Institute

\begin{tabular}{|c|c|c|}
\hline Thème & Nature des problèmes & Année \\
\hline $\begin{array}{l}\text { Remblai ferroviaire sur plage du port de } \\
\text { Xiamen }\end{array}$ & Etudes spécifiques du site pour le projet & $1982-83$ \\
\hline Rupture du quai Wuwam & Etudes géomécaniques pour la réparation & 1983 \\
\hline Consolidation d'argiles molles & Validation de modèles numériques & 1985 \\
\hline $\begin{array}{l}\text { Barrage en enrochement de Xibeikou avec } \\
\text { revêtement en béton }\end{array}$ & $\begin{array}{l}\text { Etude paramétrique et validation } \\
\text { d'études numériques pour le projet }\end{array}$ & $1986-87$ \\
\hline $\begin{array}{l}\text { Vidange rapide du réservoir d'un barrage en } \\
\text { enrochement non revêtu }\end{array}$ & Etudes pour le projet & 1987 \\
\hline $\begin{array}{l}\text { Stabilité du barrage en terre Yangmaowan } \\
\text { fondé sur du loess }\end{array}$ & Etudes pour amélioration de la stabilité & 1987 \\
\hline $\begin{array}{l}\text { Géomembrane dans le noyau en terre d'un } \\
\text { barrage en enrochement établi sur une faille } \\
\text { active }\end{array}$ & Etudes pour le projet & 1988 \\
\hline $\begin{array}{l}\text { Pentes maximales admissibles pour les épau- } \\
\text { lements d'un barrage en terre }\end{array}$ & Etudes pour le projet & 1988 \\
\hline
\end{tabular}

Tableau 3. - Liste des études géotechniques en centrifugeuse à I'Université de Huhai. Table 3. - List of the geotechnical centrifuge studies at Huhai University

\begin{tabular}{|c|c|c|}
\hline Thème & Nature des problèmes & Année \\
\hline Consolidation d'un dépôt d'argile molle & Recherche appliquée & 1982 \\
\hline Stabilité de pente & idem & 1982 \\
\hline Tassement de remblai & idem & 1982 \\
\hline $\begin{array}{l}\text { Stabilité des fondations d'une digue du port } \\
\text { de Chiwan }\end{array}$ & Etude spécifique pour un projet & 1983 \\
\hline $\begin{array}{l}\text { Stabilité d'un batardeau pour terre-plein rem- } \\
\text { blayé au port de Liangun }\end{array}$ & idem & 1984 \\
\hline Stabilité d'une écluse dans la baie Lianshi & idem & 1985 \\
\hline $\begin{array}{l}\text { Stabilité de batardeaux pour digue de l'usine } \\
\text { pétrochimique de Shanghaï }\end{array}$ & idem & 1985 \\
\hline Consolidation de sol mou sous dépression & $\begin{array}{l}\text { Etude de l'efficacité d'une méthode d'améliora- } \\
\text { tion des sols }\end{array}$ & 1984 \\
\hline $\begin{array}{l}\text { Stabilité d'une digue et d'un mur de quai au } \\
\text { port Yantai }\end{array}$ & Etude spécifique pour un projet & 1985 \\
\hline $\begin{array}{l}\text { Stabilité de barrages composés en cendres } \\
\text { volcaniques }\end{array}$ & idem & $1986-87$ \\
\hline
\end{tabular}


Tableau 4. - Liste des études géotechniques en centrifugeuse dans les autres universités et instituts chinois. Table 4. - List of the geotechnical centrifuge studies of other universities and institutes

\begin{tabular}{|c|c|c|c|}
\hline Thèmes & Nature des problèmes & Institut & Année \\
\hline Remblai routier de Daihuang sur argile molle & Etude spécifique pour projet & IRSRY & 1987 \\
\hline $\begin{array}{l}\text { Etude des contraintes pour des structures bâties sur } \\
\text { fondations non uniformes }\end{array}$ & idem & IRSRY & 1987 \\
\hline $\begin{array}{l}\text { Grand barrage en terre de Xiao-Longdi sur alluvions de la } \\
\text { rivière Jaune }\end{array}$ & idem & IRSRY & $1988-90$ \\
\hline $\begin{array}{l}\text { Barrage en enrochement sur alluvions épaisses avec } \\
\text { rideau d'étanchéité en béton }\end{array}$ & idem & ICEHC & 1985 \\
\hline Mur de soutènement & Recherche appliquée & $\begin{array}{l}\text { Univ. } \\
\text { Sud-Ouest }\end{array}$ & 1986 \\
\hline $\begin{array}{l}\text { Stabilité d'un dépôt compressible de grande épaisseur } \\
\text { à Shanghaï }\end{array}$ & Etude spécifique pour projet & ICFS & 1987 \\
\hline Capacité portante de semelles sur des sables & Etude paramétrique & $\begin{array}{l}\text { Univ. } \\
\text { Tsing Hua }\end{array}$ & 1986 \\
\hline Comportement d'un mur en sol renforcé & Recherche appliquée & $\begin{array}{l}\text { Univ. } \\
\text { Changsha }\end{array}$ & 1987 \\
\hline
\end{tabular}

\section{CONCLUSIONS}

Le développement économique de la Chine va conduire à rencontrer dans le futur un grand nombre de problèmes géotechniques. Dans ce contexte, il est certain que la modélisation en centrifugeuse a un brillant avenir. Bien que nous ayons franchi une première étape, nous devons continuellement améliorer les techniques d'essais, les méthodes de mesures, les installations... Nous sommes désireux d'apprendre de nos collègues étrangers et espérons que tous les chercheurs échangeront leur expérience. En particulier, le Comité Technique sur les essais en centrifugeuse (TC-2) de la Société Internationale doit mener la tâche d'orienter tous les pays dans les applications de la modélisation en centrifugeuse.

\section{BIBLIOGRAPHIE}

JIA P.Z., WANG W.H., RU L.A. (1988), The survey of conceptual design for a $450 \mathrm{~g}$-t geotechnical centrifuge. Centrifuge'88, Corté J.F. (éd.), pp. 17-22, Balkema, Rotterdam.

LIU L.D., TANG J.H. (1988), A huge centrifuge for geotechnical studies. Centrifuge'88, Corté J.F. (éd.), pp. 33-36, Balkema, Rotterdam.

WANG X. (1988), Studies on the design of a large scale centrifuge for geotechnical and structural testing. Centrifuge'88, Corté J.F. (éd.), pp. 23-28, Balkema, Rotterdam.

ZHU W., LIU S. (1988), NHRI geotechnical centrifuge operation. Centrifuge'88, Corté J.F. (éd.), pp. 49-54, Balkema, Rotterdam.

ZHU W., YI J. (1988), Application of centrifuge modelling to study a failed quay wall. Centrifuge'88, Corté J.F. (éd.), pp. 415-420, Balkema, Rotterdam. 